\section{JAUNDICE AMONG THE BRITISH TROOPS IN NORTHERN ITALY.}

BY H. H. TOOTH, M.D. СамB., O.B., C.M.G., LATE CONSULTING PHYSICIAN IN ITALY;

AND

E. G. PRINGLE, M.D. LoND., M.R.C.S., L.R.C.P. LoND., LATE CaPTAIN, R.A.M.C.

Note by H. H. T.

IN August, 1918, I saw with Captain H. G. Broadbridge, R.A.M.C., who wds in charge of the "Signal Schools" Hospital at Valsanzibio, about $25 \mathrm{~km}$. south of Vicenza, a group of ten cases from the signal schools camp in the mountains close by. They presented many features in common, as follows:

Onset sudden without rigor, so sudden that the exact hour of the first symptom could be given in most cases. The first symptoms were " dizziness," a general feeling of illness, and headache, at first slight but growing in severity during the succeeding two or three days; this symptom was common to all. These men were admitted on the day of onset with temperatures of between $101^{\circ}$ and $105^{\circ}$, and this fever was maintained with irregular fluctuations, but rarely falling to $100^{\circ}$, for 6-8 days, except in one fatal case in which it fell during the fourth day to $97.6^{\circ}$, and on the sixth and seventh, the day of death, to $96.6^{\circ}$. The pulse as a rule was not commensurate with the temperature; for instance, in one case the readings are T. $103 \cdot 6^{\circ}$, P. 100, R. 30 , and similar readings might be multiplied. The rapid respiration rate is suggestive of pulmonary complication, but I found no signs in those I examined. Pains general, or in the legs or back, occurred in eight. In nearly all suffusion of the conjunctivæ was noted as an early symptom and in half of them soreness or redness of the fauces, and three showed herpes labialis. Of this group only one developed moderate catarrhal lung symptoms. In no case was the spleen or liver enlarged.

In addition to these cases Captain Broadbridge writes that shortly after this 18 more were admitted from the same source, and these he considered to be cases of so-called "epidemic pyrexia."

Taken as a whole, these two groups, totalling 28 cases, from one camp presented a general symptomatic similarity to the type of influenza which had been rife among the troops and civil population for some months previously. But among them, and in their onset and symptoms indistinguishable from the rest, were six cases ( 21.4 per cent.) which developed jaundice, and as jaundice was very common at that time they seem worthy of special notice. This jaundice appeared on about the sixth or seventh day of the illness, except in the fatal case in which it occurred on the fourth. Two of these I saw on my visit, and the others developed after it, but unfortunately I was prevented by illness from following them further.

Sapper G. H. D., aged 26, suddenly became “ dizzy" and chilly at 6 P.M. on July 24 th. He was admitted the same evening, with T. $101^{\circ}$, P. 102 , and R. 22 , headache and pains in the calves, and suffusion of the conjunctivæ appeared the next day. The fever was maintained at between $101^{\circ}$ and $102 \cdot 6^{\circ}$ for four days, fell to $100^{\circ}$ on the sixth, on which day he was jaundiced without any additional symptoms except for a crop of slightly raised purpuric blotches about the left loin and abdomen. With the fall of temperature the pulse-rate became abnormally slow, 46-66. He was transferred to No. 9 C.C.S. and from there evacuated to the base. No further notes are forthcoming, but from our experience we should suspect a continuance of low-grade fever.

Driver J. H., aged 20. Sudden onset with "giddiness" at 9 A.M. on July 26 th, slight sore-throat and general pains. Admitted same day with a temperature of $102.4^{\circ}$, rising to $103.6^{\circ}$, falling suddenly to $97 \cdot 8^{\circ}$ on the morning of the third day, but rising again the same day to $101 \cdot 6^{\circ}$. On the fourth day jaundice appeared and the temperature fell from $102^{\circ}$ to $97^{\circ} 6^{\circ}$, and finally to $96^{\circ} 6^{\circ}$. The pulse-rate was $100 \mathrm{when}$ the fever was at its highest, and respirations 30 without palmonary signs. The jaundice became very intense, the tongue thickly coated white, and he suffered from repeated vomiting, intermittent headrche, and severe general illness. No physical signs referable to heart, lungs, liver, or spleen were found at any time. He died in No. 9 C.C.S. on the seventh day of his illness. The post-mortem report is as follows:- "Acute pancreatitis. All tissues and organs deeply stained. The pancreas was swollen and hæmorrhagic. Inflammatory swelling around the orifice of the bile-duct and also adhesion at the foramen of Winslow. No free fluid in the lesser sac. Liver, spleen, kidneys, and other organs apparently healthy except for bile-staining."
Most unfortunately no microscopical or bacteriological report on these organs has come from the laboratory owing to a change of pathologists at that time, and an important link which might have thrown light on this and the other milder cases is thus lost.

We have here, then, a group of 28 cases having common characters in the early. stages, as to onset and symptoms and from the same place. Some of them develop jaundice, and, judged by the course of other cases to be detailed later by E. G. P., probably ran a long course of low fever. Others, the majority, are non-icteric. The icteric forms resemble clinically the spirochætal type. Have the non-icteric cases the same infective origin? Or are all influenzal, as one at first thought, with catarrhal jaundice as a complication. If so, why should such a simple complication so materially change and lengthen the further course of the disease? Or, lastly, have we two epidemic diseases occurring coincidently?

I have but little information as to the later stages of this group, except inferential. One icteric case returned to duty in 18 days, an unusually short course. One died as reported above. Four were evacuated to the base or to France, and of these 1 only came under our observation subsequently. Of the non-icteric cases, 3 were evacuated to the base; of 12 there is no information. The remaining 7 were returned to duty from the local schools hospital or the C.O.S. after varying periods of stay in hospital-namely, 2 for three days only, 2 for 17 days, 3 for 18, 21 , and 42 days respectively-an average stay of 17 days. Most of these periods are long for the type of uncomplicating influenza then prevalent.

The special interest to us in this group lies in the first-hand information at the earliest of a type of jaundice of which we saw a considerable number of instances in the later stages at the base, cases in which this information was very meagrely recorded on the field medical card.

In order to obtain some collective information on points such as are discussed in the following analysis, Colonel Howell, A.D.M.S., L. of C., caused reports on all cases of jaundice to be returned by medical officers of all units on L. of $C$. and base area during the months of September, October, and November, which also include a few in July, August, and December. As might be expected, these returns are very variable in value, but an analysis of them brings out some information after all reservation has been made. 128 returns of cases of jaundice came to hand, of these 17 were rejected, leaving 111 for consideration.

$$
\text { Analysis of Returns. }
$$

Seasonal incidenoe.-The greater number of cases occurred in the months of September and October, 58 and 38, respectively, 96 in all. In August 16, in July 3, in November 5 , and in December 3, totalling 123 returns in which a date was given.

Regional inoidence. - The greater number of returns are of cases occurring in the forward area-i.e., in the front line 12 or just behind it 16 , the remainder being between this and Vicenza 21, and south of Vicenza 28, 2 from Padua. Thirteen came from places which cannot be identified on the map but were certainly in one of these regions, so that 92 came from the forward area ( 90 per cent.). Ten only came as isolated cases from places on the $L$. of $C$.

" Catarrhal" cases, 84 returns. - These are cases which are stated to have had no fever at any time, but included among them are three which had a very slight short rise above normal. The symptoms at onset in 41 are abdominal, pain in the epigastrium and indisposition, nausea 7 , general malaise 5, diarrhoea 2 only, headache and vomiting 13, vomiting 4,72 in all.

Onset of jaundice from the first day of illness. Fifty-eight returns are available for this point. Fifty cases developed jaundice on various days from the first to the eighth, and of these $37 \mathrm{up}$ to the fourth day, in 16 jaundice was practically the first symptom. Of these 58 cases the average day of onset was the fourth.

Where a record of the first appearance of bile in the urine is made this symptom, as might be expected, precedes the disappearance of the skin colouration by two or three days.

Date of reappearance of bile in the stools. This was noted in only 29 cases, and it is a very variable one, from the third to the twenty-first day. The average day is the twelfth, and that is probably an outside time.

Date of disappearance of icteric tinge from skin and conjunctivæ. This is a small point, but as a return was made 
in 31 the figures may be mentioned. The largest number, 20, fell between the eighteenth and twenty-seventh days. The shortest period was 8 days from the onset of jaundice, the longest 31 days, the average being 21 .

Albuminuria was recorded in 25 cases and absent in 42 in the 67 cases in which an examination was made. 44 returns are silent on this point. Spirochætes negative, microscopically by inoculation in 2.

These returns of catarrhal jaundice are given for what they are worth. They came from all parts of the war area, but mainly from the more active forward area. They present elusive clinical features contrasting in this respect with the pyrexial group now to be analysed.

\section{Analysis of Pyrexial Group.}

Jaundice with a definite pyrexial onset. - $\mathbf{A}$ comparatively small but important group of 27 cases (24 per cent. of the whole number), it includes the cases referred to in $\mathbf{E}$. G. P.'s note (infra), but not those of H. H. T.'s group in the first part of this article. The onset is noted as sudden in at least 14 of them, probably in all. In 17 the temperature is recorded as from $99^{\circ}$ to $104^{\circ}$, and in those in which it was not recorded on the field medical card the symptoms were such that a high temperature was practically certain. Among thesymptoms at onset were returned : abdominal pain, mostly epigastric, general pains, headache, epistaxis, sore-throat, vomiting, diarrhcea only in two.

Initial fall in temperature. This was noted in 17 cases, in 11 of which the fall took place on the fourth to the ninth day, the shortest period being the fourth, the longest the fourteenth day from the onset of the illness, giving as an average day the eighth. The fall of temperature, which generally marks the onset of the jaundice is followed by a short apyrexial period. This is returned in 14 cases, in 9 of which it lasted for from 2 to 6 days, the average period from these figures is 5.7 days, which is probably an outside estimation. The apyrexial interval is followed by a variable, but generally prolonged, period of irregular low grade fever, as described below by $\mathrm{E}$. G. P., 19 returns give information on this point, but in some the temperature had not become normal at the time of the return, so that the average made is probably somewhat within the truth. The shortest period of secondary fever is given as 8 days and the longest is 57, the average being 33 days.

Onset of jaundice. - This was noted in all the 27 returns. In 20 of them the day of onset was from the third to the eighth, 10 being the third or fourth. The appearance of jaundice tends to precede that of the initial fall in the temperature, but that particular point is made in fewer cases. The average day of onset is the sixth (strictly 5.9). In the catarrhal group this day would appear to be earlieri.e., fourth, average.

Reappearance of bile in the fæces dated from the onset of jaundice. As this is an indication of the duration of obstruction it is a fact of some importance, and a return was made in 19 cases, but unfortunately in about half of these the early note is defective, and it is only stated that at the time of admission to the unit in the base area the stools contained bile. The obstruction period is therefore certainly less than the figures at our command would warrant. It is disappointing that an observation so simple, and yet so interesting, should have been made in so few cases. With this reservation, in 15 the fæces were said to be bile-coloured in from 6 to 12 days after the appearance of the jaundice. An average of 8 days of obstruction for the 19 cases is probably too long, and 6 days is likely to be nearer the truth.

Disappearance of bile from the urine, noted in 16 returns to occur at all periods from the eighth to the forty-sixth day, gives an average day as the twenty-first.

Disappearance of icteric tinge in skin and conjunctivæ, mentioned in 21 returns, was complete in the average by the thirty-fifth day.

Albuminuria. - A note on the urine is made in 25 returns, information having been required as to the presence of albumin, casts, and spirochætes. Albumin is definitely stated to be present in 12 cases, and casts in 5 of them. The remainder were returned as negative. As to spirochxtes there is no evidence that they were specially searched for except in $\mathbf{E}$. G. P.'s returns, and he records a negative result in all the cases examined microscopically-i.e., 12, in 4 of which also inoculation of guinea-pigs was performed without effect.
Note by E. G. P.

This note is based upon 29 cases of jaundice under observation at a stationary hospital at the base during the months of August, September, and October, 1918. The cases were drawn from the front line, intermediate, and base areas.

They fell naturally into two divisions: 1 . The catarrhal type, in which fever was entirely absent or very slight-that is, not over $100^{\circ} \mathrm{F}$.- during the first few days of illness. Of these, there were 17, and in two cases only a record of any fever. 2. The pyrexial type, which again divided itself into two classes : (a) initial fever followed by a long-continued low grade of fever, in all seven cases; $(b)$ initial fever followed by a secondary rise of limited duration, five cases.

All the 29 cases fell within these divisions, and so far as is known there was no case which presented a high grade of initial fever which was not followed by the long-continued low grade of fever or by fever of a relapsing type. Of the 29 cases only two originated at the base itself, and these were of true catarrhal type. The others originated either in the front line itself or in the advanced lines of communication. Except for the two catarrhal cases mentioned above these cases did not arrive at the base until ten days or more had elapsed from the commencement of the illness, and therefore one had to depend upon the information to be obtained from the field cards or from the patients themselves, guided by the records on the field cards.

Although the pyrexial type is that which presents the most interesting features, it will be useful to give a description of the catarrhal cases, as it will serve as a useful contrast to the pyrexial cases, and will also be a record of catarrhal cases occurring in Northern Italy.

The catarrhal oases, then, were as a rule distinguished by a gradual onset, by absence of or only slight fever at the onset, by the tendency to early onset of jaundice, by the presence of clay-coloured stools, by the symptoms being mostly referable to the intestinal tract rather than generalised, by the rapid onset of convalescence after the disappearance of the jaundice, and by the absence of tachycardia as a sequela. The cases varied in severity from a very slight illness to one of a moderate grade of intensity. General symptoms, such as headache, dizziness, giddiness, pains in the back and limbs, cough, may be present, but they do not form the prominent features of the illness. These were referable to the intestinal tract and abdomen, and in addition to the jaundice consisted of loss of appetite, furring of the tongue, thirst, abdominal pains, nausea or vomiting, constipation or diarrhœea. Some abdominal pain was always present, but it varied very much in severity from the ordinary epigastric pain of indigestion and irregular griping abdominal pains without abdominal tenderness to constant severe pain with distension and a general abdominal tenderness, but this latter was unusual. The position of the pain or tenderness in these cases is of importance, as only five of them referred it to the region of the gall-bladder or right costal margin, the others referring it to either the epigastrium or generally to the abdomen. In addition to the symptoms mentioned above, others occasionally noted were insomnia, general weakness, pains in the chest, drowsiness. One patient had a fine petiechial rash on the chest and abdomen on the tenth day of illness, and one said that at the beginning of the illness there were streaks of blood in the vomit. None complained of itching of the skin and none had epistaxis or herpes.

The jaundice was characterised by a tendency to appear early in the course of the case. In the two cases which were local admissions the jaundice was present in both on the first day, but taking the whole 17 cases, in 11 the jaundice appeared during the first five days of the illness. The early onset of the jaundice was accompanied by a correspondingly early appearance of bile in the urine and disappearance of bile from the stools, these events generally showing themselves on the same day or the day after the appearance of the jaundice. The bile reappeared in the stools in two-thirds of the cases within two weeks, and in all within three weeks, of the onset of the illness. But disappearance of the bile from the urine was of later date, in two-thirds within three weeks and in all within four weeks.

The skin was normal in all but one within a month and was either coincident with, or followed in a few days, the disappearance of bile from the urine, but the conjunctivæ showed a tendency to remain coloured for a distinctly longer time than the skin, but only in one case for over a week.

D 2 
In all the cases when bile was present in the urine albumin was also present, and in all when the bile disappeared the albumin also disappeared. In 2 cases casts were present in the urine; in one they were hyaline in character, in the other granular.

The spleen was enlarged to percussion in 9 cases, and in two of these instances was palpable. The liver was enlarged to percussion in 6 cases, but was never palpable.

The position of the apex beat of the heart was noted in every case, as it is of importance with relation to

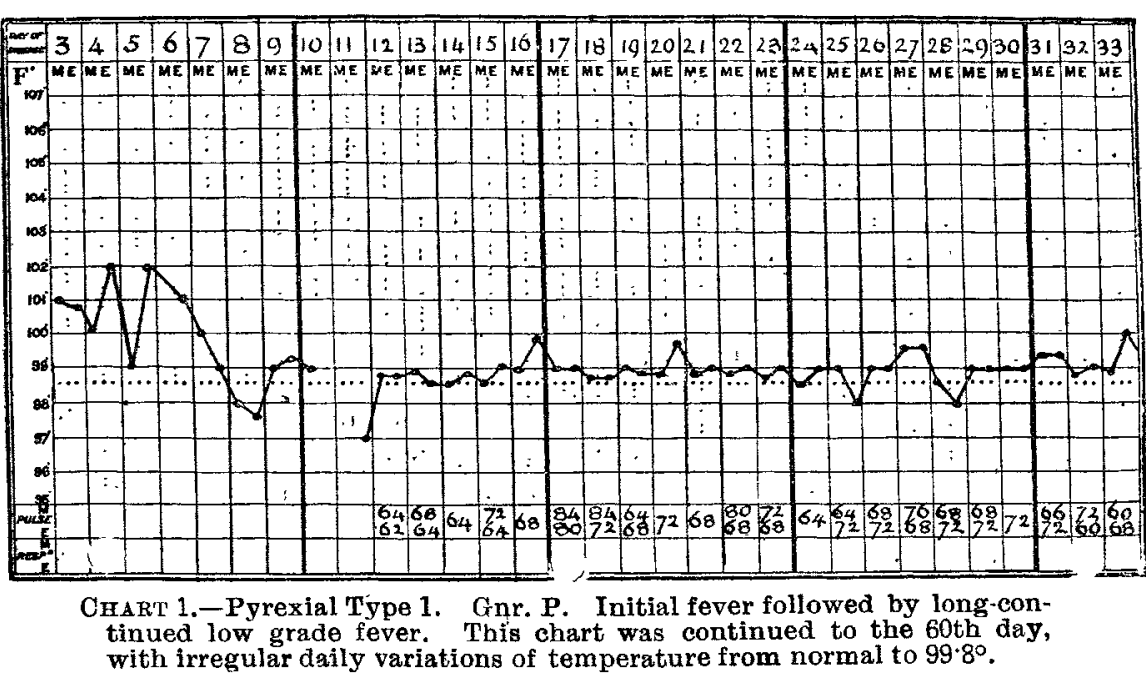

enlargement of the liver, and in 5 of the cases where the liver was enlarged to percussion the apex beat was found in the fourth left interspace. The pulse-rate in 13 of the 17 cases was abnormally slow. Two cases showed reduplication of the heart sounds.

The examination of the stools showed in 3 cases out of the 17 the presence of the ova of Triohocephalues dispar, but as these ova were very commonly found in Italy in the stools of patients of all sorts, surgical and medical, it cannot be said that they should be regarded as a cause of the jaundice when present in this proportion.

One of the cases was a convalescent dysentery, and in another the jaundice appeared while the patient was in hospital suffering from a boil of the thigh.

All cases except one were convalescent within one month of the onset of the disease.

The pyrexial cases now claim our consideration, and they fall into two classes according to the type of fever. Those with: (1) an initial fever of about seven to ten days with an interval of five to seven days, in which the temperature remains about the normal, followed by a long-continued low grade of fever which lasts from about 30 to 60 days. (Chart 1.) (2) An initial fever of about seven to ten days with an interval of five to seven days, in which the temperature remains about the normal, followed by a secondary rise of about seven days' duration, and that again sometimes followed by a short tertiary rise, after which the temperature tends to settle to the normal in distinctly less time on the average than in the first class of case. (Chart 2.)

Of the 12 pyrexial cases, there were seven in the first class and five in the second class, and apart from the type of fever and perhaps a maried lemon tinge of the skin in the first class there was very little, if any, difference in the symptoms in the two classes, although in the second class there was not the same uniformity of severity as in the first.

The seven cases with a continued fever were all acute in onset, and the attack severe and prolonged. The symptoms were those which mark the onset of an acute pyrexial attack-viz,, shivering and chill. high fever, sudden malaise, aching of the head, body, and limbs, thirst, marked weakness, sore-throat or injection of fauces, giddiness and dizziness, photophobia, suffnsion of eyes, stiffness of neck muscles, accompanied by or followed in the course of a day or two by abdominal symptoms, pains in the stomach, vomiting, constipation or diarrhoea, jaundice appearing between the fourth and seventh days, commonly on the sixth day of the illness. With the onset of jaundice there was a rapid decline of the fever, but although the patient generally felt better there was usually some drowsi. ness, apathy, very foul tongue, and bad appetite, perhaps epistaxis or a petechial rash. The jaundice rapidly became very marked and very gradually died away, but bile was absent from the urine in all the cases at least three weeks before the fever entirely died away. With the disappearance of the jaundice the skin assumed the lemon tint, and anæmia was marked in all. The lemon tint made it extremely difficult to say when the jaundice had really disappeared, as the two merged into one another. By the end of the second week the continued fever had generally started on its long and tedious course, during which it often stayed monotonously about $99^{\circ}$ for some weeks. With regard to the abdominal conditions, the local symptoms did not compare in intensity with the more general ones, but the abdomen was usually tender, sometimes swollen, and the tenderness and pain showed a marked preference for the region of the gallbladder and the right lower costal margin rather than the epigastrium. The condition of the bowels was at the onset of the illness either one of constipation or of diarrhoea, generally the former. One patient had herpes which developed on the sixth day with the jaundice, and one had swelling of the glands of the neck accompanying his sore-throat. Two had sorethroat which was of a severe character, but in neither was it the initial symptom. All the cases were extremely tedious in their con. valescence, the fever not abating in the least severe until six weeks had elapsed from the beginning of the illness, and one had fever lasting ten weeks. After the fifth week there was a marked tendency to tachycardia.

There were five cases with the second type of pyrexia. Two of these presented the same sudden onset and symptoms as the first group. A short syropsis of their symptoms is appended. These two men belonged to the same battalion and were both stationed at Arzignano. The one was taken ill a day previous to the other. They were not companions, nor was there any apparent connexion between them in their duties. Their temperature charts show a remarkable similarity; the clinical picture of their cases was also similar.

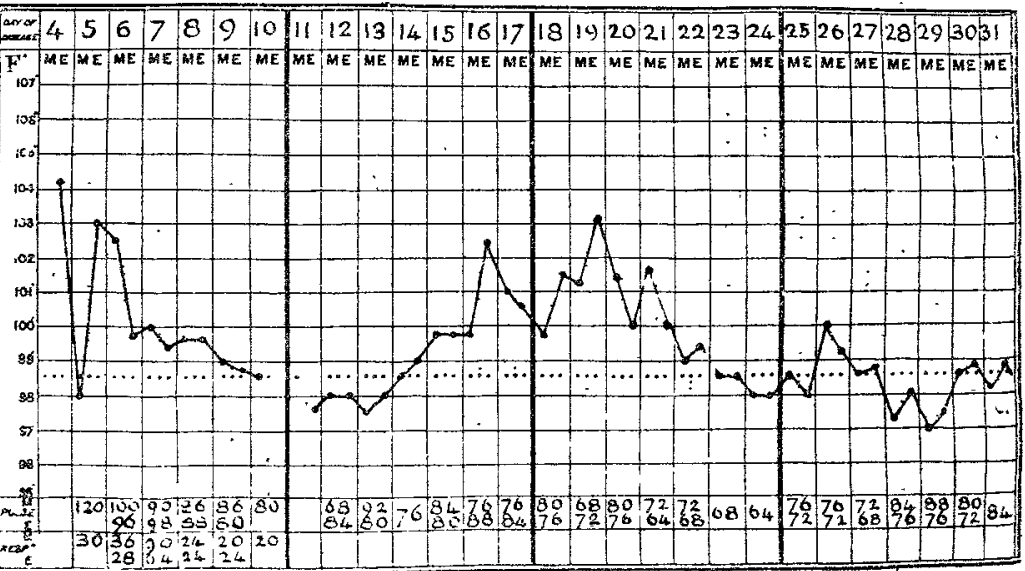

Chart 2.-Pyrexial Type 2. Case 2, Pte. D. Initial fever followed by secondary rise of limited duration.

Case 1.-Pte. I. Date of onset, 31.7.18. Onset very sudden, with sickness, diarrhcea, pains all over the body, headache and fever, pains in the stomach, and cough. 2.8.18: Jaundice and herpes. 5.8.18: Jaundice very marked, cough, headache, pains in the stomach. 11.8.18: Tongue very dirty, liver enlarged and tender, but not palpable. 14.8.18: Pain in the splenic region, with a fine friction over the spleen. No evidence of splenic enlargement. 26.9.18: Alveolar abscess. 29.9.18: Pleural friction at the right base, back and front, of a coarse character.

CASE 2.-P te. D. Date of onset, 1.8.18. Onset very sudden, with pains in the head, fever, and shivering. 2.8.18: Slight epistaxis. 3.8.18: Hæmoptysis and great weakness. 4.8.18: Temperature $104 \cdot 2^{\circ}$; jaundice. 5.8.18: Jaundice marked and pains in the stomach. 6.8.18: Herpes of lips and left cheek. 11.8.18: Deep jaundice, erythematous and urticarial 
rash of forearms, chest, and abdomen. Lower liver edge tender but not palpable. Spleen not enlarged. 13.8.18: Some small petechix in rash. 15.8.18: Rash almost disappeared. 16.8.18: Temperature $102 \cdot 4^{\circ}$; apathetic. 19.8 .18 : Tempera16.8.18: Temperature $102.4^{\circ}$; apathetic. 19.8.18: Tempera-
ture $103.2^{\circ}$; vomiting and severe pains in the back; liver and spleen not enlarged. 23.8.18: Fever gone and patient rapidly improving in general condition.

Both showed a tendency to tachycardia after the fifth week.

The other three cases included in this group did not show the same severity of symptoms, and their fever was neither so high nor so prolonged, but they all had an initial fever followed by a secondary fever of limited duration.

The question then arises, Do these two groups of pyrexial cases own the same cause? As their symptoms are so much alike and their only difference appears to be the type of fever, it seems that this question must be answered in the affirmative.

Taking, then, these pyrexial cases as one group, in the 12 cases observed, the earliest appearance of jaundice was on the third day, in 2 the jaundice appeared on the fourth, and in the remainder between the fifth and tenth days, the commonest day being the sixth. Bile appeared in the urine shortly after the onset of the jaundice, but with regard to the stools there was nothing like the same constancy of disappearance of the bile from the stools, as in the catarrhal cases. Sometimes the stools were clay-coloured, but very often some amount of bile persisted in the stools throughout the illness. Bile was present in the urine in most instances up to the fourth or fifth week. Another feature with regard to the severe types was that the jaundice improved in spite of the persistence of the fever, and in most instances the temperature was still febrile after the bile had entirely disappeared from the urine.

The skin and conjunctivæ were not generally normal until the fifth to the eighth week, and the skin and conjunctive both seemed to approach to the normal at the same time, but owing to the lemon-tinted skin in the majority of cases it was difficult to say when the bile exactly disappeared from the skin. Casts were found in the urine in 5 cases out of the 12 . In 1 hyaline, in 2 granular, and in the other 2 hyaline, granular, and epithelial. Epithelial casts were not found in the catarrhal forms. Albumin was present in the urine in all cases when bile was present. The spleen was enlarged to percussion in 5 cases, and in one of these it was palpable, this proportion not differing greatly from that found in the catarrhal cases. The liver was enlarged to percussion in 9 cases, in one of which it was palpable, the proportion here being much greater than in the catarrhal forms. The position of the apex beat of the heart was in the fourth space in 6 out of the 8 cases in which it was noted, and the liver was not enlarged in the 2 cases where it was in the normal position. The maximum point of pain or tenderness in the abdomen was in the position of the gallbladder or under the right costal margin in 6 cases out of 11 , in the epigastrium in 3 , in the splenic region in 1 , and in the left side of the abdomen in 1. This tendency for the pain to be situated in the right hypochondrium is doubtless in correlation with the greater proportion of cases of hepatic enlargement in these cases as compared with the catarrhal ones. The pulse-rate at the commencement of the illness seemed to vary, in some cases being slow in com. parison with the temperature, in others commensurate with the fever, but with the onset of the jaundice it became abnormally slow in comparison with the fever, the lowest pulse-rate noted being 32 .

The examination of the stools of these 12 cases disclosed the presence of the ova of parasitic worms in 6 of the 12, the ova of Triohoophalus dispar only in 3 , the ova of Asoaris lumbricoides only in 1, the ova of Trichocephalus aispar and ascaris in 1, and the ova of Triohooephalus dispar and an adult ascaris in 1 . As has been observed previously, these ova were quite commonly found amongst the troops in Italy, but the proportion found in these cases seemed to be above the average.

A blood count taken from Pte. D. on the seventeenth day of the disease during the secondary rise of temperature showed 4,096,000 red cells and 7000 white cells with nothing distinctive in the differential count. Blood pressure estimations showed nothing abnormal.

Five of the pyrexial cases were examined as to the presence of the' enteric group of organisms in urine and freces, three of the first group and two of the second. In all of these enteric group organisms were absent.
Blood cultures were taken from Pte. D. and Pte. L., the first being negative and the second showing a growth of Staphylococous aureus, probably a contamination.

Investigations were undertaken as to the presence of the Spirochata ioterohemorrhagia. In four of the cases, two of each group of the pyrexial types, a guinea-pig was inoculated but without result. All cases, both pyrexial and catarrhal, had a microscopical examination made of the urine, but the spirochæte was never found. Nevertheless, in spite of these negative results as to the presence of the spirochrte, it is felt that this may be a possible cause of the pyrexial cases, as they agree in their clinical character with the disease so well desoribed by Dawson, Hume, and Bedson in their paper on spirochrtal jaundice, although they do not specifically describe cases with low continued fever. There were no deaths among these 29 cases.

Conclusion.-During the months of August, September, and October, 1918, there were prevalent amongst British troops in Northern Italy two types of jaundice-namely, catarrhal and pyrexial. The pyrexial cases again were subdivided into two groups, those with initial fever followed by a long low continued fever, and those with initial fever followed by a secondary fever of limited duration. These pyrexial cases apart from the temperature presented the same clinical features and showed a great resemblance to cases of spirocbrtal jaundice, but there was no bacteriological evidence to support this view.

I must add my indebtedness to Lieutenant-Colonel $\mathrm{C}$. Bramhall, R.A.M.C., for allowing these cases to be under my care, and to Major W. Broadbent, R.A.M.C., for his suggestions and interest in these cases.

\section{Conoluding Remarks.}

We regret that by the force of circumstances the clinical facts in this communication are not supported by more bacteriological evidence. The bacteriological examinations were made by Captain W. Broughton-Alcock and Captain A. N. Smith, to whom we are much indebted, and whose technique is beyond question. The inoculations on guinea-pigs were made on the sixteenth, seventeenth, eighteenth, and twentyseventh days of the disease, respectively, and therefore within the recognised limit of 28 days, but were all negative as to spirochætal infection.

We are forced, therefore, to suspect that there may be some infection other than spirochætal, though clinically similar to it, and that, if so, all these cases, with or without jaundice, may have this infection as a common causal factor. Nevertheless, we do not feel able to exclude dogmatically the spirochætal factor, in spite of negative bacteriological results, in the face of the clinical resemblance of our cases to those published by the Japanese and British observers.

Although jaundice may occur as a complication of influenza, it is certainly not common in most civil epidemics, yet it is striking that in our first group these icteric cases should be so intimately associated in origin with, and so similar in their early symptomatology to, what seem to be "epidemic pyrexial" cases, and this circumstance gives an added interest to epidemic fever or influenza which raged in Italy from the early months and onwards of 1918.

We conclude with offering our thanks to the A.D.M.S. L. of C., Colonel Howell, for coöperation in obtaining returns, and the medical officers of all units for furnishing them.

LIVINGSTONE COLLEGE.-Dr. T. Jays, who has worked in different capacities with the Church Missionary Society and the Student Christian Movement, has become vice-principal at Livingstone College, and with his wife will reside in the College. In addition to helping the principal, Dr. Jays will take the lectures previously delivered by Colonel G. B. Price, who is now working with the Ministry of Pensions. Before the College reopens on Oct. 1st a short course of 15 lectures on Personal Care of Health in the Tropics will be given from Sept. 22nd-25th by the principal, vice-principal, and probably Colonel Price. These lectures are open to men and women, and are intended for those who expect to reside or travel in the tropics. They would be of use to missionaries, nurses, explorers, members of the services, Government officials, commercial men, \&c. Application for terms and conditions of attendance should be made previous to the lectures to the Principal, Livingstone Ccllege, Leyton, E. 10, who will be pleased to answer any questions with reference to these lectures or concerning the fall nine months' course at Livingstone College, which will commence on Wednesday, Oct. Ist, 1919. 